\title{
The power of one
}

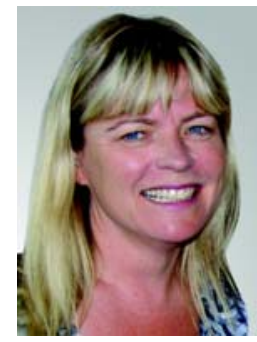

Ruth Armstrong Senior Deputy Editor

mja@mja.com.au

doi: 10.5694/mjal3.c0603 core value of clinical medicine is to do the very best for every single patient. But the principles of evidence-based practice involve developing a management plan that is informed by studies showing the net result of an intervention is balanced towards benefit. Accordingly, some patients will be helped by it, some will experience a null effect, and others will be harmed. How can we reconcile this with the practitioner's focus on each individual patient's interests? This question, though not novel, is implicit in many clinical decisions but is rarely articulated.

A current prominent example is the contention over whether to screen men for prostate cancer. Several articles in this issue revisit this question. Martin and colleagues' analysis (page 546) concludes that prostate-specific antigen screening is likely to only be cost-effective in the $<1 \%$ of men classified as very high risk. While this is valuable information, it begs the question of how to identify these high-risk men. From a purely clinical perspective, the balance between the benefits and harms of prostate cancer screening remains unresolved, despite recent large studies, because of disagreement about how to interpret the evidence, say Del Mar and colleagues (page 525).

Nevertheless, they offer welcome advice for walking individuals through the maze of uncertainty - but suggest that the conversation only be had at the patient's request.

In response, Hugosson and Carlsson cite their own study, which showed a survival benefit from screening that was largely offset by an almost equivalent loss of qualityadjusted life-years, mainly from the effects of treatment (page 528).

These can be difficult concepts for doctors, let alone patients. But how do we make a decision about screening for any cancer? According to Rychetnik and colleagues (page 536), our thinking should go beyond benefits, harms and costs, to include social and ethical aspects. They say that "we need to ensure accountability to the citizens whose lives are changed, for better and for worse, by cancer screening programs".

With regard to vaccination programs - an area of disease prevention with far less ambiguous outcomes - high participation rates are necessary for success. Here, doctors can be confident that the benefits outweigh the harms in spades. Yet when adverse events (rarely) occur, rapid and accurate ascertainment is vital. Clothier and colleagues' useful study (page 554) will inform postlicensure surveillance after this year's roll-out of human papillomavirus vaccination for adolescent boys. Using knowledge from the existing program for girls, they estimated the background rates of neurological and allergic events that might be potentially associated with vaccination in boys, to establish a benchmark for early detection if more than the expected number of events should occur.

Clinical guidelines assist in bringing population-level evidence to bear on individual patient management. However, they are open to interpretation and, even when fully applied to a management plan, not every patient will have the best possible outcome. A father recounts his 29-year-old daughter's delayed diagnosis of advanced and ultimately fatal colorectal cancer (page 562), and asks the Gastroenterological Society of Australia (GESA) to review its recommendations for investigating iron deficiency anaemia in young women, to advise earlier colonoscopy in severe cases. On behalf of GESA, Sievert and colleagues (page 563) reply that GESA recommendations would advise investigation of this patient's persistent anaemia, but that the minimal yield from colonoscopy in a very low-risk population for colorectal cancer should be balanced against the procedure's risks.

"Nightmare" clinical cases like this do not make for comfortable reading. They also give the lie to medicine being solely guided by rigid decision points, protocols and flowcharts. Doctors' seamless use of the one-on-one relationship with each of their patients - to give context to population-level evidence for the most appropriate management plan — will always be a cornerstone of clinical care. After all, the benefits to patients of having someone versed in the evidence, but relentlessly supporting their interests, far outweigh any harms.

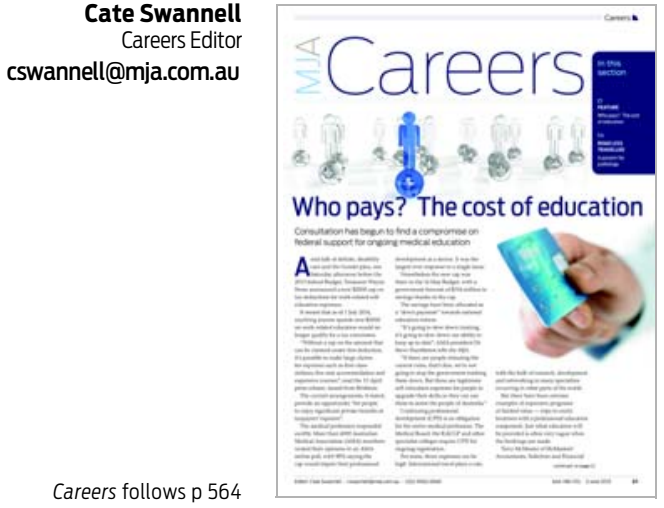

\section{Who pays?}

On a Saturday afternoon before the 2013 federal Budget, Treasurer Wayne Swan announced a new $\$ 2000$ cap on tax deductions for work-related selfeducation. As of 1 July 2014, anyone who spends over $\$ 2000$ on workrelated education will no longer qualify for a tax concession. Annabel
McGilvray spoke with Australian Medical Association president Dr Steve Hambleton and others about the impact the cap will have on Australian doctors (pages C1, C2). In Road less travelled, Professor Ibrahim Zardawi shares his passion for pathology with Cate Swannell (page C4). 
Editor's choice

516 MJA $198(10) \cdot 3$ June 2013 